\title{
Concomitant new diagnosis of systemic lupus erythematosus and COVID-19 with possible antiphospholipid syndrome. Just a coincidence? A case report and review of intertwining pathophysiology
}

\author{
Eduardo Mantovani Cardoso ${ }^{1}$ (D) $\cdot$ Jasmin Hundal $^{1} \cdot$ Dominique Feterman $^{1} \cdot$ John Magaldi $^{2}$
}

Received: 14 June 2020 / Revised: 20 July 2020 / Accepted: 23 July 2020 / Published online: 28 July 2020

(C) International League of Associations for Rheumatology (ILAR) 2020

\begin{abstract}
In the midst of the COVID-19 pandemic, further understanding of its complications points towards dysregulated immune response as a major component. Systemic lupus erythematosus (SLE) is also a disease of immune dysregulation leading to multisystem compromise. We present a case of new-onset SLE concomitantly with COVID-19 and development of antiphospholipid antibodies. An 18-year-old female that presented with hemodynamic collapse and respiratory failure, progressed to cardiac arrest, and had a pericardial tamponade drained. She then progressed to severe acute respiratory distress syndrome, severe ventricular dysfunction, and worsening renal function with proteinuria and hematuria. Further studies showed bilateral pleural effusions, positive antinuclear and antidouble-stranded DNA antibodies, lupus anticoagulant, and anticardiolipin B. C3 and C4 levels were low. SARS-Cov-2 PCR was positive after 2 negative tests. She also developed multiple deep venous thrombosis, in the setting of positive antiphospholipid antibodies and lupus anticoagulant. In terms of pathophysiology, COVID19 is believed to cause a dysregulated cytokine response which could potentially be exacerbated by the shift in Th1 to Th2 response seen in SLE. Also, it is well documented that viral infections are an environmental factor that contributes to the development of autoimmunity; however, COVID-19 is a new entity, and it is not known if it could trigger autoimmune conditions. Additionally, it is possible that SARS-CoV-2, as it happens with other viruses, might lead to the formation of antiphospholipid antibodies, potentially contributing to the increased rates of thrombosis seen in COVID-19.
\end{abstract}

Keywords Antiphospholipid syndrome · Autoimmunity · COVID-19 - Cytokine release syndrome - Systemic lupus erythematosus

\section{Introduction}

Systemic lupus erythematosus (SLE) is a multisystem autoimmune disease caused by a complex interplay of genetics and environmental exposure with various possible triggers [1]. Coronavirus disease 19 (COVID-19) is an infection caused by a novel coronavirus SARS-CoV-2, which became a pandemic in 2020 after an initial outbreak in Wuhan, China [2].

Eduardo Mantovani Cardoso mantovanicardoso@uchc.edu

1 Internal Medicine Residency, University of Connecticut School of Medicine, Farmington, CT, USA

2 Hartford Hospital, Hartford, CT, USA
Early reports from COVID-19 pathophysiology suggest that an overt inflammatory response, akin to cytokine release syndrome, could be a major contributing factor in the morbidity and mortality [3]. It is known that both SLE and APLS can be triggered by viral illnesses [4-6]; however, there is very little literature to suggest that COVID-19 could be potentially associated with SLE presentation, although that was already theorized in the literature [7].

Patients with SARS-CoV-2 infection show a severe inflammatory cytokine release storm which leads to high expression of pro-inflammatory cytokines, such as IL-1, IL-6, and TNFalpha, that leads to clinical presentations similar to autoimmune diseases [7]. It is well documented that environmental triggers, such as viral infection, lead to the activation of innate and acquired immune response in genetically predisposed patients. Therefore, one can suspect that genetically predisposed 
patients show viral susceptibility, and this can lead to the development of rapid autoimmune dysregulation causing hyper-inflammatory autoimmune diseases [7].

We present a catastrophic case of new-onset SLE with possible antiphospholipid syndrome and concomitant COVID-19 in an 18-year-old female. We also discuss the pathophysiological basis for a possible association between those entities and implications for therapy.

\section{Case description}

An 18-year-old Hispanic female with a past medical history of autism spectrum disorder and panic disorder presented to an outside hospital (OSH) due to a 2-day history of productive cough, shortness of breath, and fevers superimposed on a month's history of upper respiratory symptoms and overall malaise. In the OSH, she was noted to be tachypneic, tachycardic, and hypotensive, with subsequent deterioration into cardiac arrest. ROSC was achieved after $5 \mathrm{~min}$. Pointof-care ultrasound in the emergency department showed pericardial effusion with tamponade physiology. Emergent pericardiocentesis was performed with drainage of $400 \mathrm{cc}$ of amber colored fluid (exudate- $3.6 \mathrm{~g} / \mathrm{dL}$ protein, LDH $275 \mathrm{U} /$ L) with subsequent improvement in hemodynamics. Initial labs were significant for serum creatinine $(\mathrm{SCr})$ of $2.0 \mathrm{mg}$ / $\mathrm{dL}$, blood urea nitrogen (BUN) of $49 \mathrm{mg} / \mathrm{dL}$, white blood cell (WBC) count of $10.500 / \mu \mathrm{L}$ ( $80 \%$ neutrophils), hemoglobin $9.5 \mathrm{~g} / \mathrm{dL}$, and platelets $242.000 / \mu \mathrm{L}$. Arterial blood gas initially showed a $\mathrm{pH}$ of $7.2, \mathrm{PaCO} 2$ of $35 \mathrm{mmHg}$, and $\mathrm{PaO} 2$ of $63 \mathrm{mmHg}$ on $100 \%$ of fraction of inspired oxygen (FiO2). Chest X-ray showed multifocal airspace consolidation, predominantly in the middle and lower lung fields and bilateral pleural effusions. Follow-up transthoracic echocardiography showed left ventricular ejection fraction (LVEF) of 20-25\% with left ventricle dilatation and no regional wall motion abnormalities.

She remained on mechanical ventilation and later developed severe acute respiratory distress syndrome (ARDS) with a $\mathrm{PaO} 2 / \mathrm{FiO} 2$ ratio of 78 , requiring very high positive end expiratory pressure (PEEP), up to 20, on high FiO2 (80$100 \%$ ). She tested negative for SARS-CoV-2 with PCR-RT, 4 days apart. Viral panel was otherwise negative. Of note, she developed lymphopenia with lowest absolute lymphocyte count of 0.3 thousand $/ \mu \mathrm{L}$.

Over the course of a week, her renal function deteriorated, and she developed anuria requiring hemodialysis (HD). As this progressed, further studies were performed. Urinalysis showed proteinuria (urine protein/creatinine ratio 2.84) and hematuria, and serologies were positive for antinuclear antibodies (1:2560), antidouble-stranded DNA, and low complement levels. Additionally, the patient was found to have anticardiolipin antibodies as well as positive lupus anticoagulant. All those findings led to a diagnosis of SLE (ACR/ EULAR 2019 score of 22) (Table 1).

During her stay at the OSH, she received pulse steroids for 3 days, 4 days of ceftazidime, vancomycin, azithromycin, and hydroxychloroquine. She was then transferred to our service for ECMO evaluation and higher level of care.

Upon admission, she was continued on steroids and underwent one session of plasmapheresis as a second line therapy for severe manifestations of SLE. Furthermore, there was still high clinical suspicion for COVID-19, and the patient was retested for a third time with PCR-NAAT, which came back positive. Then, plasmapheresis was discontinued due to a concern of possible removal of antibodies needed for the adaptive response to SARS-CoV-2. She was managed supportively for severe ARDS, including pronation for 3 days. She received 1 dose of tocilizumab as part of our hospital protocol for severe COVID-19 with elevated inflammatory markers. She also developed severe anemia and thrombocytopenia (respective nadirs $5.4 \mathrm{~g} / \mathrm{dL}$ and 32.000), thought to be multifactorial, which resolved with supportive care and transfusions. She then developed multiple DVTs, raising concerns for antiphospholipid syndrome, and was started on anticoagulation.

She went into pulseless electrical activity arrest on combined day 17 of hospitalization and unfortunately passed away.

\section{Discussion}

Systemic lupus erythematosus is a multisystem, autoimmune disease characterized by systemic inflammation and tissue damage. The disease is an interplay between the immune system, hormones, and the environment, as well as genetic susceptibility $[1,4,8]$. Our patient presented with a new diagnosis of SLE, possible APLS, and concomitant COVID-19. There are no reported cases, thus far, that we are aware of, of a patient diagnosed with both COVID-19 and new-onset SLE, although there were reports of an association between antiphospholipid syndrome and COVID-19 [9].

In this patient's case, there was overlap between findings consistent with SLE and COVID-19, such as myopericarditis and pulmonary compromise. Prior data showed that pericardial effusion is common in SLE, found in $10-54 \%$ of patients [10], while it is rare in COVID-19, subject of case reports [11]. Cardiac tamponade is uncommon but reported in both conditions [12-15]. Additionally, she had left ventricle dilation, reduced EF, global hypokinesis, and troponin elevation (peak $0.8 \mathrm{ng} / \mathrm{dL}$ ), which, in the setting of pericardial effusion, likely suggests myopericarditis that could be seen in both SLE and in COVID-19 [10, 16]. 
Pulmonary manifestations are common in SLE, with pleural effusion being the most common one, affecting almost $50 \%$ of patients during their lifetime [17]. On the other hand, in COVID-19, pleural effusions are uncommon, roughly seen in about $2 \%$ of patients [18]. The patient also had multifocal airspace consolidation on chest X-ray, which could be seen in $34 \%$ of SLE patients with pulmonary manifestations [17]. In comparison, in COVID-19, this imaging modality shows bilateral pneumonia appearance in about $70 \%$ of patients [19]. Our patient was not submitted to a high-resolution

Table 1 Laboratory values

\begin{tabular}{|c|c|c|c|}
\hline General & Labs at admission & Peak/nadir values & Other relevant labs \\
\hline Hemoglobin (g/dL) & 9.5 & 5.4 (day 2) & \\
\hline Platelets (units/ $\mu \mathrm{l}$ ) & 242.000 & 32.000 & \\
\hline White blood cell count (thousand/ $\mu$ l) & 10.2 & 38.6 & \\
\hline Absolute lymphocyte count (thousand/ $\mu$ ) & 1.5 & 0.3 (day 4 ) & \\
\hline Creatinine $(\mathrm{mg} / \mathrm{dL})$ & 2.0 & 4.6 (day 8 ) & \\
\hline BUN (mg/dL) & 49 & 82 (day 17 ) & \\
\hline $\mathrm{ABG}-\mathrm{p} . \mathrm{H} / \mathrm{PaO} 2 / \mathrm{PaCO} 2(\mathrm{mmHg})$ & $7.2 / 35 / 63$ on $100 \%$ FiO2 & & \\
\hline Creatinine kinase $(\mathrm{U} / \mathrm{L})$ & 564 & & \\
\hline \multicolumn{4}{|l|}{ Inflammatory } \\
\hline Ferritin $(\mu \mathrm{g} / \mathrm{L})$ & Not performed & 2112 (day 16) & \\
\hline $\mathrm{LDH}(\mathrm{U} / \mathrm{L})$ & 751 & 805 (day 8) & \\
\hline $\mathrm{CRP}(\mathrm{mg} / \mathrm{dL})$ & 0.94 & 3.73 (day 8$)(<0.49$ reference value) & \\
\hline $\operatorname{ESR}(\mathrm{mm} / \mathrm{h})$ & Not performed & 38 (day 7$)$ & \\
\hline \multicolumn{4}{|l|}{ Coagulation } \\
\hline D-dimer (ng/mL DDU) & $1784(<230)$ & 2443 (day 11) & \\
\hline Fibrinogen $(\mathrm{mg} / \mathrm{dL})$ & $169(148-435)$ & & \\
\hline INR & 1.1 & 1.7 & \\
\hline PTT (s) & 32 & 54 & \\
\hline Dilute Russell viper venom (s) & & 85 & \\
\hline Drvvt 1:1 mix (seconds) & & 59 & \\
\hline Drvvt confirmation & & Positive, day 6 & Negative, day 8 \\
\hline Anticardiolipin IgA (U/mL) & & $12.1(<12$ reference value $)$ day 6 & Negative days 8,13 \\
\hline Anticardiolipin IgM/IgG (U/mL) & & Not performed day 6 & Negative days 8.13 \\
\hline Antibeta-2-glycoprotein $1 \mathrm{IgM} / \mathrm{IgG}$ & & Not performed day 6 & Negative day 8 \\
\hline \multicolumn{4}{|l|}{ Immunology } \\
\hline Antinuclear antibody (HEp-2) & & $>=1: 2560$, homogeneous pattern & \\
\hline Antidouble-stranded DNA (IU/mL) & & $943(<4$ reference value $)$ & \\
\hline Antihistone & & & Positive \\
\hline Anti-SSA & & Negative & \\
\hline Anti-SSB & & Negative & \\
\hline Antismith & & Negative & \\
\hline Rheumatoid factor & & Negative & \\
\hline Anti-RNP antibody & & Negative & \\
\hline c-Anca & & Negative & \\
\hline p-Anca & & Negative & \\
\hline Antimitochondrial M2 & & Negative & \\
\hline $\mathrm{C} 3$ (mg/dL) & & $29(83-193)$ & \\
\hline $\mathrm{C} 4(\mathrm{mg} / \mathrm{dL})$ & & $9(15-57)$ & \\
\hline Urine protein/creatinine ratio & & & 2.84 \\
\hline Urine hemoglobin & & & Positive \\
\hline Urine RBCs & & & Too many to count \\
\hline Beta-2-microglobulin & & & 16.9 (1.21-2.70 reference value) \\
\hline
\end{tabular}


computerized tomography of the chest, whose findings would have been useful in differentiating those entities.

Regarding demographics in COVID-19, less than $1 \%$ of patients requiring ICU level of care were younger than 20 years old, and about $5 \%$ had less than 40 years of age with unreported prevalence of autoimmune diseases [2, 20]. Of note, our patient had 2 negative SARS-CoV-2 PCR tests and was positive on the third test, which could be explained by the reported sensitivity for nasopharyngeal swab being around $70 \%$ and operator dependent for an adequate specimen collection [21,22]. Although we cannot exclude that she could also have acquired COVID-19 while hospitalized, literature is scarce on hospital-acquired COVID-19.

Generally, SLE patients are at an increased risk of infections due to the dysfunction in the innate and adaptive immune systems because of the development of autoantibodies, immune complexes, and impaired clearance of apoptotic and necrotic material [4]. In this context, COVID-19 generates a robust immune response. The $\mathrm{S}$ protein of coronavirus binds to the host cells via the angiotensin-converting enzyme 2, ACE2, fuses with the membrane, and releases the viral RNA [23]. The ACE2 receptor is found in the lower respiratory system, which leads to an inflammatory response in the lower lung airways. This causes a downstream initiation of pathways which can activate cytokines and chemokines such as IL-1, IL-2, IL-4, IL-7, IL-10, IFN-gamma, and TNF- alpha [3, 23]. This cytokine storm is thought to be central in this disease process that leads to acute respiratory distress syndrome and multi-organ failure [3, 23].

The adaptive immune system in patients with SLE is typically impaired and could be possibly further compromised in the infection with the novel coronavirus. Various studies have shown increased autoreactivity of helper T cells, cytotoxic T cells, B cell differentiation, and autoantibody production in SLE [8]. This leads to impairment in the production of interferon gamma, IL-1, IL-2, and TNF alpha, compromising a Th1 type of response, which is, generally, more effective than a Th2 response against viral infections $[8,24]$. The change in the cytokine production with the shift from the Th1 to Th2 cells has been described with HIV infection, and this could be a potential explanation in COVID-19 leading to the occurrence of autoimmune phenomenon and potentially disease. Prior reports also found an association between Epstein-Barr virus, parvovirus B19, retrovirus, and cytomegalovirus and the pathogenesis of autoimmune diseases $[4,5,8]$. Those factors might explain why the patient had severe COVID-19 and an unfavorable outcome. As a side note, both the patient's mother and grandmother had only mild COVID- 19 .

Additionally, the patient was found to have anticardiolipin A antibodies, lupus anticoagulant, and thrombocytopenia. While in the ICU, she developed multiple DVTs and was started on anticoagulation. This clinical presentation raised concern for APLS. Although it was not possible to fulfill the modified Sapporo criteria as the patient unfortunately passed away before 12 weeks for repeat testing, two explanations are possible. First, she might have developed APLS in the setting of SLE [25]. Second, transient lupus anticoagulant and antiphospholipid antibodies are known to develop in the setting of viral infections, and a growing body of literature suggests that COVID-19 could also be associated with these findings $[6,9]$.

Although recent data has shown that COVID-19 predisposes to both venous and arterial thromboembolisms, the exact pathophysiology involved in this hypercoagulable state is not clear. However, there seems to be a severe inflammatory state that causes not only the life-threatening pneumonia but also a severe derangement of hemostasis [26]. While it was initially hypothesized that patients were hypercoagulable from acute disseminated intravascular coagulation (DIC), others believe that a profound derangement of hemostasis and endothelial dysfunction might be the etiology [26]. In addition to that, a case series reported that COVID-19 was associated with antiphospholipid antibodies and thrombosis in multiple vascular territories [9]. This could explain partially why as many as $31 \%$ of critically ill patients in the ICU with COVID-19 were found to have thrombotic complications [27].

Infectious agents have been shown, in vivo, to trigger the formation of antiphospholipid antibodies through molecular mimicry between bacteria or viruses and the beta2glycoprotein-I (such as its lipid-binding site), especially in genetically susceptible patients, potentially leading to a prothrombotic state and fetal wastage [28, 29]. These patients can have both venous and arterial thrombi, most commonly lower extremity DVTs and ischemic strokes [28]. This could be through either the direct formation of thrombi or activation of endothelial cells, platelets, and other cells activating the coagulation cascade [28]. Viruses have been associated with formation of anti-beta-2-glycoprotein antibodies, which could be more strongly related to the characteristic manifestations of APLS $[6,29,30]$. Nonetheless, this could explain in part our patient's multiple DVTs, as well as the increased incidence of thrombotic complications in patients with COVID-19, and could guide clinicians who are diagnosing and managing future cases with similar thromboembolic complications. Furthermore, additional research is needed to determine if there is a relationship between antiphospholipid antibodies and hypercoagulability in COVID-19, including better understanding of how SARS-CoV-2 might induce the generation of these antibodies in predisposed individuals.

Different therapeutic approaches targeting the cytokine storm, such as IL-6 and IL-1 inhibitors, corticosteroids, and JAK2 inhibitors, are postulated as possible treatments for COVID-19 patients by blunting the dysregulated immune response [31-33]. The patient received high dose steroids as she had an indication for that from the SLE standpoint. She also 
received Plaquenil and tocilizumab which were being used as compassionate drugs at that point. Further research on various medications for COVID-19 acting in the cytokine release syndrome is ongoing to answer if that might lead to better outcomes for patients.

\section{References}

1. Tsokos GC (2011) Systemic lupus erythematosus. N Engl J Med 365(22):2110-2121. https://doi.org/10.1056/nejmra1100359

2. Wang D et al (2020) Clinical characteristics of 138 hospitalized patients with 2019 novel coronavirus-infected pneumonia in Wuhan, China. Jama 323(11):1061. https://doi.org/10.1001/jama. 2020.1585

3. Qin C et al (2020) Dysregulation of immune response in patients with COVID-19 in Wuhan, China. Clin Infect Dis. https://doi.org/ $10.1093 / \mathrm{cid} / \mathrm{ciaa} 248$

4. Doaty S et al. (2016) "Infection and lupus: which causes which?" Curr Rheumatol Rep, vol. 18, no. 3, doi:https://doi.org/10.1007/ s11926-016-0561-4.

5. Perl A (1999) Mechanisms of viral pathogenesis in rheumatic disease. Ann Rheum Dis 58(8):454-461. https://doi.org/10.1136/ard. 58.8 .454

6. Uthman IW, Gharavi AE (2002) Viral infections and antiphospholipid antibodies. Semin Arthritis Rheum 31(4):256263. https://doi.org/10.1053/sarh.2002.28303

7. Caso F et al (2020) Could SARS-Coronavirus-2 trigger autoimmune and/or autoinflammatory mechanisms in genetically predisposed subjects? Autoimmun Rev 19(5):102524. https://doi. org/10.1016/j.autrev.2020.102524

8. Esposito $\mathrm{S}$ et al (2014) Infections and systemic lupus erythematosus. Eur J Clin Microbiol Infect Dis 33(9):1467-1475. https://doi. org/10.1007/s10096-014-2098-7

9. Zhang Y et al. (2020) "Coagulopathy and antiphospholipid antibodies in patients with Covid-19.” N Engl J Med, vol. 382, no. 17, doi:https://doi.org/10.1056/nejmc2007575.

10. Tincani A et al (2006) Heart involvement in systemic lupus erythematosus, anti-phospholipid syndrome and neonatal lupus. Rheumatology 45(suppl_4):iv8-iv13. https://doi.org/10.1093/ rheumatology/kel308

11. Inciardi RM et al (2020) Cardiac involvement in a patient with coronavirus disease 2019 (COVID-19). JAMA Cardiol. https:// doi.org/10.1001/jamacardio.2020.1096

12. Castier MB et al (2000) Cardiac tamponade in systemic lupus erythematosus. Report of Four Cases. Arq Bras Cardiol 75(5):446448. https://doi.org/10.1590/s0066-782x2000001100008

13. Kahl LE (1992) The spectrum of pericardial tamponade in systemic lupus erythematosus: report of ten patients. Arthritis Rheum 35(11): 1343-1349. https://doi.org/10.1002/art.1780351115

14. Dabbagh MF et al (2020) Cardiac tamponade secondary to COVID-19. JACC: Case Rep. https://doi.org/10.1016/j.jaccas. 2020.04.009

15. Hua A et al (2020) Life-threatening cardiac tamponade complicating myo-pericarditis in COVID-19. Eur Heart J. https://doi.org/10. 1093/eurheartj/ehaa253

16. Kang $Y$ et al (2020) Cardiovascular manifestations and treatment considerations in Covid-19. Heart. https://doi.org/10.1136/heartjnl2020-317056
17. Alamoudi OSB, Attar SM (2015) Pulmonary manifestations in systemic lupus erythematosus: association with disease activity. Respirology 20(3):474-480. https://doi.org/10.1111/resp.12473

18. Wu J et al (2020) Interpretation of CT signs of 2019 novel coronavirus (COVID-19) pneumonia. Eur Radiol. https://doi.org/10.1007/ s00330-020-06915-5

19. Rodriguez-Morales AJ et al (2020) Clinical, laboratory and imaging features of COVID-19: a systematic review and meta-analysis. Travel Med Infect Dis 34:101623. https://doi.org/10.1016/j.tmaid. 2020.101623

20. Grasselli G et al (2020) Baseline characteristics and outcomes of 1591 patients infected with SARS-CoV-2 admitted to ICUs of the Lombardy Region, Italy. Jama. https://doi.org/10.1001/jama.2020. 5394

21. Wang $\mathrm{W}$ et al (2020) Detection of SARS-CoV-2 in different types of clinical specimens. Jama. https://doi.org/10.1001/jama.2020. 3786

22. Xie X et al. (2020) "Chest CT for typical 2019-NCoV pneumonia: relationship to negative RT-PCR testing." Radiology, p. 200343. doi:https://doi.org/10.1148/radiol.2020200343

23. Guo Y-R et al. (2020) "The origin, transmission and clinical therapies on coronavirus disease 2019 (COVID-19) outbreak - an update on the status." Mil Med Res, vol. 7, no. 1, doi:https://doi.org/10. 1186/s40779-020-00240-0.

24. Cytokines and immunity to viral infections. Ramshaw IA, Ramsay AJ, Karupiah G, Rolph MS, Mahalingam S, Ruby JC Immunol Rev. 1997; 159():119-35.

25. Garcia D, Erkan D (2018) Diagnosis and management of the antiphospholipid syndrome. N Engl J Med 378(21):2010-2021. https://doi.org/10.1056/nejmra1705454

26. Panigada $\mathrm{M}$ et al (2020) Hypercoagulability of COVID-19 patients in intensive care unit. A report of thromboelastography findings and other parameters of hemostasis. J Thromb Haemost. https://doi.org/ $10.1111 /$ jth. 14850

27. Klok FA et al (2020) Incidence of thrombotic complications in critically Ill ICU patients with COVID-19. Thromb Res. https:// doi.org/10.1016/j.thromres.2020.04.013

28. Schreiber K et al. "Antiphospholipid syndrome." Nat Rev Dis Primers, vol. 4, no. 1, 2018, doi:https://doi.org/10.1038/nrdp. 2017.103.

29. Asherson RA (2003) Antiphospholipid antibodies and infections. Ann Rheum Dis 62(5):388-393. https://doi.org/10.1136/ard.62.5. 388

30. Martirosyan A et al. (2019) "Environmental triggers of autoreactive responses: induction of antiphospholipid antibody formation." Front Immunol, vol. 10, doi:https://doi.org/10.3389/fimmu.2019. 01609.

31. Channappanavar R et al. (1970) "Why tocilizumab could be an effective treatment for severe COVID-19?" J Transl Med, BioMed Central, translational-medicine.biomedcentral.com/ articles/10.1186/s12967-020-02339-3.

32. Mehta P et al (2020) COVID-19: consider cytokine storm syndromes and immunosuppression. Lancet 395(10229):1033-1034. https://doi.org/10.1016/s0140-6736(20)30628-0

33. Zhao M (2020) "Cytokine storm and immunomodulatory therapy in COVID-19: role of chloroquine and anti-IL-6 monoclonal antibodies." Int J Antimicrob Agents, p. 105982., doi:https://doi.org/ 10.1016/j.jjantimicag.2020.105982.

Publisher's note Springer Nature remains neutral with regard to jurisdictional claims in published maps and institutional affiliations. 